Research Paper

\title{
Exosomes Derived from Human Induced Pluripotent Stem Cells-Endothelia Cells Promotes Postnatal Angiogenesis in Mice Bearing Ischemic Limbs
}

Meng Ye*, Qihong Ni*, Haozhe Qi*, Xin Qian, Jiaquan Chen, Xiangjiang Guo, Maoran Li, Yiping Zhao, Guanhua Xue, Haoyu Deng ${ }^{\bowtie}$, Lan Zhang ${ }^{\circledR}$

Department of Vascular Surgery, RenJi Hospital, Shanghai Jiaotong University School of Medicine, Shanghai, China

*These authors equally contribute to this work.

$\triangle$ Corresponding authors: Lan Zhang, Department of Vascular Surgery, RenJi Hospital, Shanghai Jiaotong Unviersity School of Medicine, Shanghai, China, 200127, Email: rjzhanglan@sjtu.edu.cn, Phone:(+86)189-1835-7825, Fax: (021)6838-5664 and Haoyu Deng, Department of Vascular Surgery, RenJi Hospital, Shanghai Jiaotong Unviersity School of Medicine, Shanghai, China, 200127, E-mail:eric.deng@hli.ubc.ca, Phone: :(+86)139-1896-9092, Fax: (021)6838-5664

(C) Ivyspring International Publisher. This is an open access article distributed under the terms of the Creative Commons Attribution (CC BY-NC) license (https://creativecommons.org/licenses/by-nc/4.0/). See http://ivyspring.com/terms for full terms and conditions.

Received: 2018.07.09; Accepted: 2018.09.26; Published: 2019.01.01

\begin{abstract}
Induced pluripotent stem cell (iPSC) derived endothelial cells (ECs) is a novel therapeutic option for ischemic diseases. Although the detailed mechanism of this novel therapy remains unknown, emerging evidence has demonstrated that exosomes derived from hiPSC-ECs play a critical role in this approach. In this study, we first isolated and characterized the exosomes from iPSCs-ECs (hiPSC-EC-Exo) and determined the functional roles of hiPSC-EC-Exo in neovascularization and the underlying mechanism. Further, we evaluated the effect of exosomes derived from hiPS-ECs on promoting angiogenesis in a mouse model bearing ischemic limbs. Our results showed that miR-199b-5p, an miRNA highly associated with angiogenesis, is significantly upregulated during the differentiation of hiPSC-ECs. Mechanically, our studies found that hiPSC-ECs expressing miR-199b-5p significantly promote cell migration, proliferation and tube formation through Jagged-1-dependent upregulation of VEGFR2 in HUVECs. Similarly, coculture of hiPSC-ECs-Exo with HUVECs also resulted in a significant improvement in HUVEC migration, proliferation, and tube formation, suggesting that exosome-mediated cell-cell communication in a paracrine manner may serve as a fundamental mechanism for iPSC-EC-based treatment. Consequently, we found that the transfer of hiPSC-ECs enriched with miR-199b-5p significantly enhanced micro-vessel density and blood perfusion in ischemic limbs in vivo. Taken together, our studies were the first to demonstrate that transfer of hiPSC-ECs-Exo is a promising approach to treat ischemic injury via the mechanism of promoting neovascularization.
\end{abstract}

Key words: hiPSC-EC, exosome, angiogenesis, Jagged-1, miR-199b-5p

\section{Introduction}

Lower extremity peripheral artery disease (PAD), especially atherosclerotic disease, is a leading cause of peripheral artery obstruction and eventual limb loss. Clinically, PAD is usually undiagnosed, mainly because of the high prevalence of asymptomatic PAD. Only severe cases present with a variety of symptoms and signs indicative of extremity ischemia. Epidemiologically, PAD affects 8 million people in the United States and over 200 million population worldwide, representing a major threat to human and public health ${ }^{1}, 2$. Current treatment options are primarily aimed at recanalization via angioplasty and stent implantation, however, restenosis and reocclusion are significant intermediate and long-term limitations in providing durable patency for PAD ${ }^{1,3}$. Thus, there is an urgent need for the development of new strategies.

Since the discovery of induced pluripotent stem cells (iPSCs) in 20064, advanced preclinical progress has been achieved by recognizing that paracrine factors generated from transplanted iPSCs, rather than the direct effects of the cells, impart the major beneficial effects of regeneration within the injured tissues in $\operatorname{PAD}^{5,6,7,8}$. Therefore, these findings have shifted our focus to the exosomes, cell-derived vesicles that contains mRNA, microRNA (miRNA) and proteins 9,10 . It is documented that iPSC-derived exosomes promote endogenous repair and enhance 
cardiac function after myocardial infarction?. Also, evidence is emerging that iPSC-derived exosomes protect $\mathrm{H} 9 \mathrm{C} 2$ cardiac cells from $\mathrm{H}_{2} \mathrm{O}_{2}$-induced oxidative stress by inhibiting caspase $3 / 7$ activation ${ }^{7}$. These results together raised the possibility that iPSC-derivatives might serve as a source of autologous bioactive exosomes for PAD treatment.

miRNAs are small non-coding RNAs which are highly expressed in endothelial cells, and recent data suggest that they regulate aspects of vascular development and angiogenesis ${ }^{11}, 12,13$. MiRNAs are secreted actively via exosomes that protect them from degradation by RNases, indicating that miRNAs may function outside the cell in which they were produced as a paracrine factor $14,15,16$. In the context of PAD, miRNA-199b is an important modulator in a myriad of vascular biology events ${ }^{17}$. Evidence has implicated that miRNA-199b in iPSC-derived endothelial cell (EC) differentiation by modulating vascular endothelial growth factor (VEGF) expression via targeting Notch signaling 17,18 . We therefore propose a novel approach using miR-199b as a potential regulator of the phenotypic switch for neovascularization via delivery of iPSC-derived exosomes enriched with miR-199b-5p, a member of the miRNA-199b family that is highly associated with angiogenesis.

Our study demonstrated that human induced pluripotent stem cell-derived endothelial cell exosomes (hiPS-EC-Exos) enriched with miR-199b-5p significantly promote neovascularization in a hind limb ischemia mouse model. In vitro experiments demonstrated that miR-199b-5p positively regulates human umbilical vein endothelial cell (HUVEC) proliferation and migration via transcriptional upregulation of vascular endothelial growth factor receptor 2 (VEGFR2). Our study also looked in depth into the mechanism and found that VEGFR2 overexpression is regulated through Jagged1/Notch1 signaling pathway. Taken together, our study is the first to reveal that adoptive transfer of hiPSC-EC-Exos enriched with miR-199b-5p is a promising approach for PAD treatment.

\section{Materials and methods}

\section{Cell culture and differentiation}

HUVECs were purchased from American Type Culture Collection (PCS-100-010, ATCC, Manassas, VA, USA). HUVECs were cultured in RPMI (\#11875 093, Gibco, Carlsbad, CA, USA) supplemented with 10\% FBS (\#10099141, Gibco, Grand Island, NY, USA), $100 \mathrm{IU} / \mathrm{ml}$ penicillin, and $100 \mu \mathrm{g} / \mathrm{ml}$ streptomycin. hiPSCs kept in our laboratory were cultured in Matrigel-coated (\#354277, BD Biosciences, Sparks, MD, USA) plates with mTeSR medium (\#85850, Stem
Cell Technologies, Vancouver, BC, Canada). The process of cell differentiation was performed as previously described ${ }^{19}$. Briefly, differentiation will be induced two days after passaging colonies by replacing TeSR-E8 medium with differentiation media based on a-MEM (Gibco) and timed addition of the following factors: $25 \mathrm{ng} / \mathrm{ml}$ Activin A (PeproTech, AF-120-14E), $30 \mathrm{ng} / \mathrm{ml}$ bone morphogenetic protein (BMP) 4 (PeproTech, AF-120-05ET), 50 ng/ml VEGF 165 (R\&D Systems, 293-VE), and the small molecule inhibitor CHIR99021 (Selleck, S1263). On day 3 and day 7 of differentiation, the medium will be refreshed with a-MEM containing $50 \mathrm{ng} / \mathrm{ml}$ VEGF and 10 $\mu \mathrm{mol} / \mathrm{L}$ SB43152 (PeproTech, 1614) only. Single-cell suspensions were incubated with PE-conjugated anti-human CD31 and CD34 antibodies, and flow cytometry was used to purify the ECs.

\section{Flow cytometry}

Purified ECs were washed with PBS twice, followed by incubation with FITC-conjugated CD31/ CD34 (\#130-117-390/\#130-113-740, MiltenyiBiotec, Bergisch Gladbach, Germany) for 30 mins in the dark. After incubation, the samples were analyzed by flow cytometry (\#653118, GE Healthcare, Piscataway, NJ, USA) .

\section{Exosome collection and quantification}

Exosome collection was performed by ultracentrifugation as previously described ${ }^{7}$. Briefly, conditional medium (RPMI medium supplemented with $4.5 \mathrm{~g} / \mathrm{L}$ glucose and $2 \mathrm{mM}$ L-glutamine) was collected and processed for gradient centrifugation at $300 \mathrm{~g}$ for $10 \mathrm{~min}, 2000 \mathrm{~g}$ for $10 \mathrm{~min}$ and $10,000 \mathrm{~g}$ for 30 $\mathrm{min}$, respectively, to obtain purified supernatants. The resultant supernatants were then used for ultracentrifugation at $100,000 \mathrm{~g}$ for $70 \mathrm{~min}$ to pellet exosomes and contaminating proteins. After washing with PBS, the pellets were processed for an additional ultracentrifugation at $100,000 \mathrm{~g}$ for $70 \mathrm{~min}$ to harvest exosomes. The size and concentration of exosomes were measured by NanoSight NS300 (Malvern Instruments, Malvern, Worcestershire, UK).

\section{Electron microscopy of exosomes and exosome labeling}

Purified exosomes were re-suspended in PBS and fixed with $2 \%$ paraformaldehyde for $30 \mathrm{~min}$ at room temperature and then dropped onto electron microscopy grids that had been pretreated with UV light to reduce static electricity. After drying for 30 min, exosomes were stained with $1 \%$ uranyl acetate twice (6 min each). The dried grids were examined using HT7700 transmission electron microscope (Hitachi, Tokyo, Japan) at $120 \mathrm{kV}$. The fluorescent dye PKH26 (\#PKH26GL, Sigma-Aldrich, Saint Louis, MO, 
USA) was used to label exosomes. Purified exosomes were incubated with PKH26 for 5 mins at $37{ }^{\circ} \mathrm{C}$, followed by washing with PBS and ultracentrifugation at $170,000 \mathrm{~g}$ for $90 \mathrm{~min}$.

\section{Cell migration assay}

The trans-well system (\#3470, Corning Inc, Tewksbury, MA, USA) was employed for cell migration assays as previously described ${ }^{20,21.1 \times 10^{4}}$ HUVECs with RPMI were seeded in the upper chambers. The medium was then replaced with fresh RPMI containing $10^{8} / \mathrm{ml}$ hiPSC-EC-Exos, $10^{8} / \mathrm{ml}$ Inhibitory-miR199b-5p-Exos, or control medium. The lower chamber was filled with 500ul RPMI containing $10 \%$ FBS. After incubation at $37^{\circ} \mathrm{C}$ for $24 \mathrm{~h}$, the cells on the upper surface were removed with a cotton swab and the cells on the undersides of the membranes were stained with hematoxylin. The number of the cells was counted under a light microscope in five random visual fields $(\times 200)$.

\section{Wound-healing assay}

HUVECs were seeded in a six-well plate at a density of $1 \times 10^{5}$ cells/ well. Sterile $10 \mu$ pipette tip was used to generate scratch wounds, and floating cells were removed by washing with PBS. Images of the scratches were taken at $0 \mathrm{~h}$ and $8 \mathrm{~h}$ of incubation using an inverted microscope at $\times 100$ magnification. The percentage of healed wound area was measured as a ratio of occupied area to the total area.

\section{Tube formation assay}

Tube formation assay was performed as previously described ${ }^{21}$. Collagen gels were formed by adding BiocoatMatrigel (\#356234, Becton Dickinson, Franklin Lakes, NJ, USA) into 24-well plates and incubating in a $\mathrm{CO}_{2}$-free incubator at $37^{\circ} \mathrm{C}$ for $30 \mathrm{~min}$. The gels were then overlaid with $5 \times 10^{4}$ cells suspended in culture medium and incubated at $37^{\circ} \mathrm{C}$ in an atmosphere of $5 \% \mathrm{CO}_{2}$. Gels were examined by using a phase-contrast microscope equipped with a digital camera PDMC (Polaroid, Minnetonka, Minnesota, USA). Images were captured and the number of branch points and tubule lengths (defined as those exceeding $200 \mu \mathrm{m}$ in length) were quantified in five randomly-chosen fields.

\section{Cell proliferation}

CCK8 kit (HY-K0301-500T, MCE, NJ, USA) was used to assess cell proliferation. In brief, cells were seeded into 96-well plates with 4000 cells per well. CCK8 reagent was added into each well at $1 \mathrm{~d}, 2 \mathrm{~d}, 3 \mathrm{~d}$, $4 \mathrm{~d}$ and $5 \mathrm{~d}$ following the experiment. Cell growth curves were mapped with time as the horizontal ordinate and OD as the longitudinal ordinate.

\section{Immunostaining}

Cells were fixed with $4 \%$ paraformaldehyde for $15 \mathrm{~min}$ at room temperature and then probed with primary antibodies: Nuclei were stained with DAPI (\#D1306, Invitrogen, Paisley, UK). An epifluorescence microscope (\#DM4000 M, Leica, Wetzlar, Germany) was used for imaging analysis.

\section{Establishment of hind-limb ischemia mouse model and laser Doppler perfusion studies}

Hind-limb ischemia (HLI) mouse model was established by ligation of femoral artery in C57/BL6J mice. HLI mice were randomly assigned to receive either intramuscular injection of PBS or exosomes injection $\left(1 \times 10^{8} / \mathrm{ml}\right)$ by direct injection of a total $20 \mathrm{ul}$ volume into 4 different sites of the ischemic hind limb immediately after surgery. Additional treatment was performed twice a week thereafter. Blood perfusion was monitored at day $0,7,14,21$ after initiation of the experiment. All animal experiments followed the protocol approved by the Animal Care Committee at Renji Hospital, School of Medicine, Shanghai Jiaotong University.

\section{Capillary density quantification}

Muscle tissue harvested from ischemic limb on day 21 was fixed in $4 \%$ methanol, paraffin-embedded, and cross-sectioned for histological immunostaining. CD31 (CD31, Abcam) was used as a mark of EC differentiation. CD31 positive sections were quantified from three independent sections from each group. CD31 positive cells from ten high power field images per condition were counted and averaged. Values are reported as the ratio of capillary density in the ischemic to non-ischemic limb. $\mathrm{P}<0.05$ was considered statistically significant.

\section{Exosomal RNA isolation and quantitative RT-PCR}

Total RNA was extracted from exosomes using an exosomal RNA extraction kit (101Bio, CA, USA) according to the manufacturer's instructions. The resulting RNA was prepared with the RNeasy mini kit (\#74104, QIAGEN, Leipzig, Germany) and treated with DNase (\#1064143, QIAGEN, Leipzig, Germany). $1 \mathrm{mg}$ of RNA was reverse-transcribed into cDNA via Oligo (dT) with miRNA qRT-PCR Detection Kit (\#A2030A004, Biotnt, Shanghai, China). Real-time quantitative PCR was performed in triplicate with SYBR Green SuperMix (\#170-8880, Bio-Rad, Hercules, California). GAPDH and Rnu6 were used as endogenous house-keeping controls. PCR primer sequences are provided in Table 1. 
Table 1. PCR primer sequences

\begin{tabular}{lll}
\hline Primer & Forward & Reverse \\
\hline $\begin{array}{l}\text { Mir-199b } \\
\text {-5p }\end{array}$ & 5'CCCAGTGTTTAGACTATCTG & 5'AACTGGTGTCGTGGAG \\
Rnu6 & 5'CAAATTCGTGAAGCGTT & 5'TGGTGTCGTGGAGTCG \\
VEGF & 5'TGTGCCCACTGAGGAGTC & 5'CATTTGTTGTGCTGTAGGAA \\
Ang-1 & 5'TGCCATTCTGACTCACATAG & 5'CAGTTGCCATCGTGTTCT \\
Ang-2 & 5'GAAAGAATGTGGCAGATTGT & 5'GGCAGGAGGAAAGTGTAG \\
IL-8 & 5'TCAGAGACAGCAGAGCACAC & 5'TTAGCACTCCTTGGCAAAAC \\
TGF-b & 5'ACTACTACGCCAAGGAGGTC & 5'GAGAGCAACACGGGTTCAG \\
bFGF & 5'GAAGAGAGAGGAGTTGTGTC & 5'AAGAAACACTCATCCGTAAC \\
VEGFR2 & 5'AGTTGGTGGAACATTTGG & 5'TACAGGAAACAGGTGAGGTA \\
GAPDH & 5'GGCCTCCAAGGAGTAAGACC & 5'AGGGGAGATTCAGTGTGGTG \\
\hline
\end{tabular}

\section{Western blot analysis}

Cells were harvested using RIPA lysis buffer (\#P0013B, BiYunTian, Shanghai, China) in the presence of halt protease and phosphatase inhibitor cocktail (\#4906845001/\#4693116001, Roche, Basel, Switzerland). Western blot analysis was performed as previously described. In brief, equal amounts of proteins were resolved by SDS-PAGE and then transferred to nitrocellulose membranes. The resulting membranes were incubated with primary antibodies at $4^{\circ} \mathrm{C}$ overnight, followed by incubation with HRP-conjugated secondary antibodies at room temperature for $1 \mathrm{hr}$. The immunoreactive bands were visualized by enhanced chemiluminescence. Primary antibodies used in this study were: Jagged-1 (\#70109, CST, Boston, MA, USA), NICD (\#3608, CST, Boston, MA, USA), HES1 (\#11988, CST, Boston, MA, USA), VEGFR2 (\#9698, CST, Boston, MA, USA), $\beta$-actin (\#4970, CST, Boston, MA, USA).

\section{Lentiviral particle transfection and drug treatment}

Lentiviral particles were produced using pre-miR-199b, inhibit-miR-199b-5p and non-targeting vector, respectively (Jima, Shanghai, China). For lentiviral infection, cells were incubated with pre-miR-199b, inhibit-miR-199b-5p or non-targeting control $(5 \times 108 \mathrm{TU} / \mathrm{ml})$ in the medium supplemented with $10 \mathrm{mg} / \mathrm{ml}$ of polybrene for 24 hours. The medium was then replaced by fresh medium supplemented with puromycin (\#A1113802, Life Technologies, Carlsbad, CA, USA)for another $48 \mathrm{hr}$. For drug treatments, DAPT (Sigma-Aldrich, B1793) was applied for the inhibition of Notch1. HUVEC cultured in serum-free medium were incubated with Notch1 inhibitor, DAPT $(75 \mu \mathrm{M})$, until the end of experiments.

\section{Statistical analysis}

All results presented are representative of at least 3 independent experiments. Results are expressed as mean \pm SD. Statistical analysis was conducted using unpaired Student's $t$ test. Values of $p<0.05$ were considered to be statistically significant.

\section{Results \\ iPSC-EC differentiation, identification and expression profile of $\mathrm{miR}-199 \mathrm{~b}-5 \mathrm{p}$}

We first performed ultracentrifugation experiment to purify iPSC-EC. As Figure 1A demonstrated that iPSC-EC initially aggregate as a cell mass, followed by divergent growth and manifestation of a typical EC morphology. In order to further identify whether iPSC-ECs differentiate to have a phenotype of ECs, immunofluorescence staining was performed to determine the presence of CD31 and CD34, which are considered as the markers of EC phenotype. Figure 1B showed that the majority of iPSC-ECs were positively stained with both CD31 and CD34, indicating that iPSCs differentiation into iPSC-ECs is achieved. Further, flow cytometry was conducted to quantify the number of positive iPSC-ECs. As Figure 1C showed, CD31 and CD34 were positively stained in $84 \%$ and $68.5 \%$ of the iPSC-ECs, respectively, indicating that the efficiency of the differentiation procedure performed in this study is superior to that of other procedures. Considering that the miR-199b$5 p$ expression profile changes during the process of iPSC-EC differentiation, we performed qPCR to evaluate the transcriptional level of miR-199b-5p at different time points of iPSC-EC differentiation. Figure 1D demonstrated that the miR-199b-5p level is significantly upregulated at day 7 and day 10 after the initiation of iPSC-EC differentiation. Moreover, Figure 1E showed that expression level of miR199b-5p in hiPSC-EC after microbeads purification is also significantly upregulated, suggesting that miR-199b$5 p$ is enriched in the cells with a phenotype of EC.

\section{miR-199b-5p promotes cell migration and proliferation}

In order to determine the functional role of miR-199b-5p in HUVEC proliferation and migration, we first established a stable HUVEC cell line expressing inhibitory miR-199b-5p using lentivirus transfection of pGMLV-MI7-has-miR-199b-5p-Inhibitor-Puro. HUVECs expressing miR-199b-5p from lentivirus transfection of pGMLV-CMV-has-miR199b-EF1-ZsGreen1-T2A-Puro were used as a positive control. The transcriptional level of miR-199b-5p was evaluated by qPCR. The results showed that miR-199b-5p was significantly downregulated in stable HUVECs expressing inhibitory miR-199b-5p as compared to HUVEC expressing stable scramble control and wild-type HUVEC, while miR-199b-5p was significantly upregulated in stable HUVECs 
expressing miR-199b-5p (Figure 2A\&B). We next performed tube-formation and transwell assays to evaluate cell migration. Figure 2C-E demonstrated that the tube formation ability of HUVECs expressing miR-199b-5p was significantly increased as compared to that of HUVECs expressing inhibitory miR-199b-5p and wild-type HUVECs, suggesting that miR-199b-5p plays a positive role in supporting cell migration. The reduction in proliferating ability of stem cells with aging hampers the regenerative ability of stem cells. In order to determine whether HUVECs enriched with miR-199b-5p have an increased ability to proliferate, we conducted a cell proliferation assay. As Figure 2F demonstrated, HUVECs enriched with miR-199b-5p have a significantly increased cell proliferation rate as compared with HUVECs enriched with inhibitory miR-199b-5p and wild-type HUVECs.

\section{hiPSC-EC-Exo supports HUVEC migration and proliferation}

Recent studies have indicated that stem cells exert their therapeutic effect via a paracrine mechanism other than transdifferentation, and exosomes are one of the most important paracrine factors for stem cells to control the reprogramming of injured cells. In order to determine whether exosomes derived from hiPSC-EC have the potential to promote HUVEC migration and proliferation, western blots for CD9, CD63 and TSG101 (exosome markers) were first performed to verify that exosomes were successfully extracted from hiPSC-ECs (Figure 3A). SEM and Nanosight results demonstrated a typical morphology of exosomes and the expected diameter of exosomes, further indicating that exosome extraction was successful (Figure 3B-C). qPCR results confirmed that the transcriptional level of miR-199b-5p in exosomal RNA of hiPSC-ECs was significantly upregulated in hiPSC-ECs as compared to inhibitory hiPSC-ECs (Figure 3D). Immunofluorescence staining of inhibitory iPSC-EC showed the presence of CD31 and CD34, indicating a endothelial phenotype of inhibitory hiPSC-ECs (Figure 3E). Next, we co-cultured hiPSCEC-Exo with HUVECs and examined the ability of HUVECs migration and proliferation. The results showed that HUVECs cocultured with hiPSC-EC-Exo enriched with miR-199b-5p have a significantly increased cell migration rate and tube-formation ability as compared to HUVECs cocultured with inhibitory-miR199b-5p and the corresponding control (Figure 3F-I). Similarly, cell proliferation assays demonstrated that HUVECs cocultured with hiPSCEC-Exo with enriched miR-199b-5p had a significantly increased proliferation rate as compared to HUVECs cocultured with inhitiory-miR199b-5p and the corresponding control (Figure 3J). Collectively, these results indicate that exosomes derived from hiPSC-EC-Exo enriched with miR-199b-5p is a potential paracrine factor that enhances the migration and proliferation of HUVEC.
A

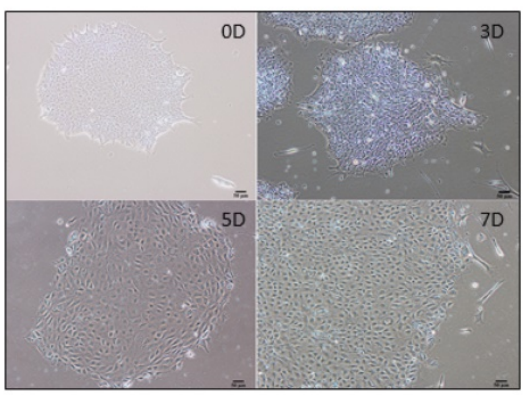

B

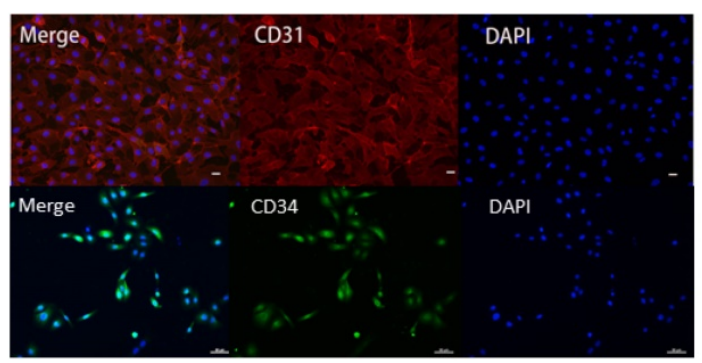

C

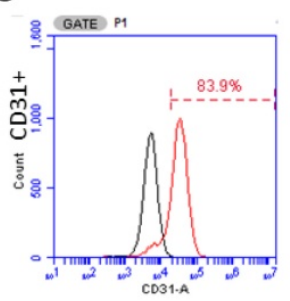

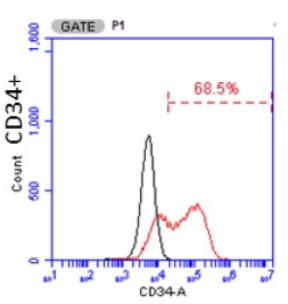

D

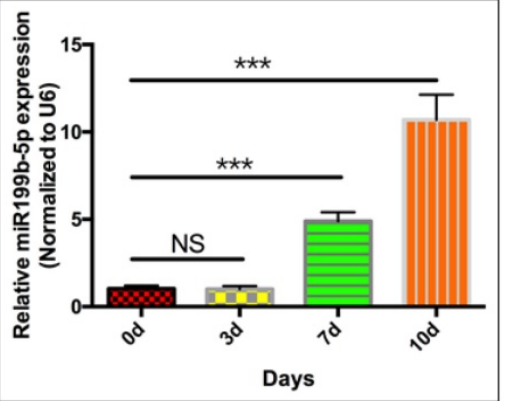

E

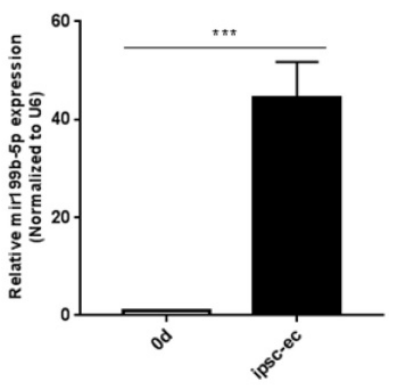

Figure 1. Generation and characterization of iPSC-EC. (A): Representative images of typical EC morphology (original magnification, $\times 100)$; (B): Immunostaining of iPSC-EC-associated markers (CD31 and CD34). Counter-staining with DAPI (original magnification, $\times 100)$; (C): FACS analysis using EC markers (CD31 and CD34); (D): miR-199b-5p expression profile of iPSC-EC. Transcriptional level of miR-199b-5p was examined by qPCR after 7 days and 10 days of initiation of the experiments. (E): Transcriptional level of miR-199b-5p in iPSC-EC was examined by $q P C R$ after microbeads purification. 


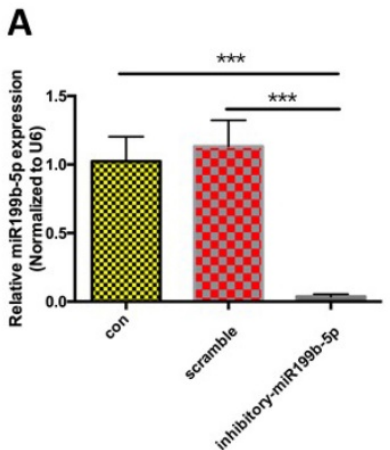

D

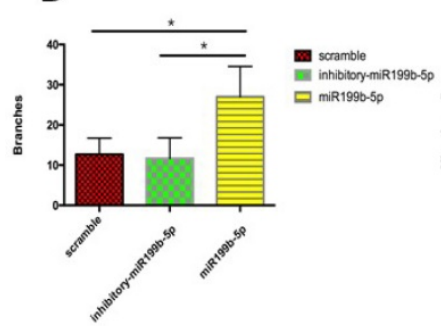

B

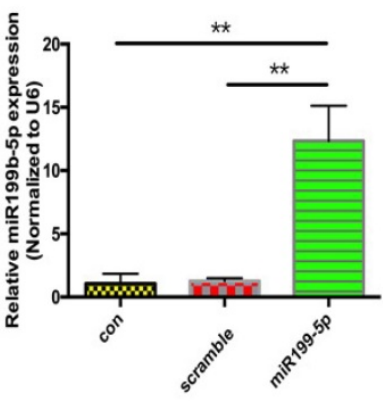

E

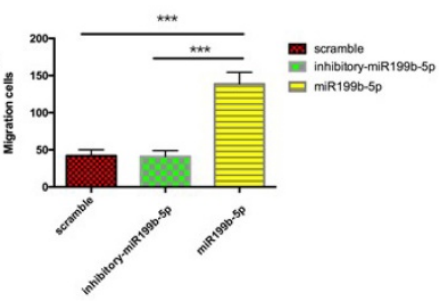

C
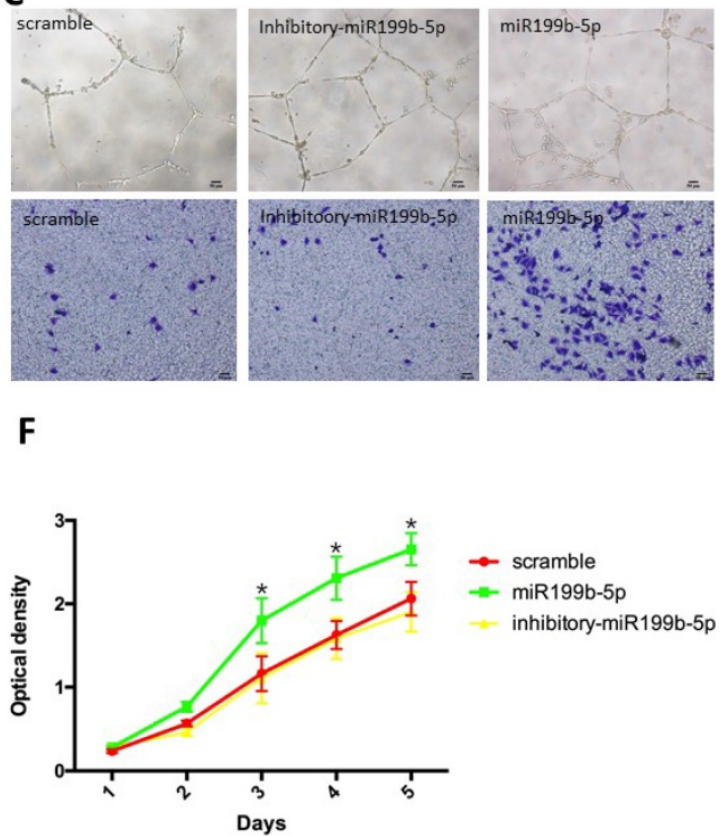

HUVECS

Figure 2. The functional role of miR-199b-5p in cell migration and proliferation. (A\&B): MiR-199b-5p expression profile of HUVEC. Transcriptional levels of miR-199b-5p in HUVECs expressing miR-199b-5p (A) or HUVECs expressing inhibitory miR-199b-5p (B) were examined by qPCR; (C) Endothelial tube formation of HUVECs cultured on Matrigel was captured by light microscopy (original magnification, $\times 100$ ); Photo images of Transwell analysis of HUVECs that stably express either miR-199b-5p or inhibitory-miR-199b-5p; (D) The tube-like structures were determined by pixel density, values are mean \pm SD, * $p<0.05$; (E) Migration rates of HUVECs that stably express either miR-199b-5p or inhibitory-miR-199b-5p, results are presented as mean \pm SD, $*{ }^{*}<0.05$; (F) Growth curves of HUVECs stably expressing either miR-199b-5p or inhibitory-miR-199b-5p were conducted by CCK- 8 assay. The OD value at $450 \mathrm{~nm}$ represents the viable cell number. Results are presented as mean \pm SD, * $\mathrm{p}<0.05$.

\section{hiPSC-EC-Exo promotes angiogenesis in vivo}

Next, we evaluated potential effects of hiPSCEC-Exo on angiogenesis in vivo. Blood perfusion of ischemic limbs was examined after treatment of hiPSC-EC-Exo. Figure 4A\&B demonstrated that treatment of hiPSC-EC-Exo had significantly improved blood perfusion of ischemic limbs from day 14 after initiation of the experiment, suggesting that hiPSCEC-Exo promotes blood perfusion in vivo. We further harvested muscle tissues from ischemic limbs for the evaluation of neovasculation density. The results revealed that treatment of hiPSC-EC-Exo significantly improved neovasculation density by manifesting an increased number of CD31 positive cells, as compared to vehicle and inhibitory-hiPSC-EC-Exo treatments, indicating that hiPSC-EC-Exo promotes angiogenesis in vivo (Figure $4 \mathrm{C} \& \mathrm{D}$ ).

\section{hiPSC-EC-Exo enriched with miR-199b-5p promotes angiogenesis via inhibition of Jagged 1/Notch 1 signaling}

Angiogenesis is a biological process controlled by the net balance between molecules that have positive and negative regulatory activities. We thus examined the mechanism by which hiPSC-EC-Exo enriched with miR-199b-5p promotes angiogenesis.
First, we determined mRNA levels of angiogenic genes, including Ang-1, Ang-2, VEGF, VEGFR2, $\beta$-FGF, TGF- $\beta$ and IL-8, between HUVECs expressing either miR-199b-5p or inhibitory miR-199b-5p. qPCR results revealed that the transcriptional level of VEGF R2 was significantly increased in HUVECs expressing miR-199b-5p as compared with that expressing inhibitory miR-199b-5p, indicating that miR-199b-5p positively regulates VEGFR2 transcription (Figure 5A). Further, we studied the mechanism by which miR-199b-5p upregulates VEGFR2 transcription. It is well-documented that Jagged1/ Notch1 signaling is required for the induction of arterial-cell fate and sprouting angiogenesis. Consistent with previous findings, our results found that inhibition of Notch1 in HUVECs significantly enhanced sprouting angiogenesis by demonstration of increased tube-formation ability (Figure 5B-D). Moreover, we found that inhibition of Notch1 significantly upregulated protein expression of VEGFR2, indicating that Jagged1/ Notch1 signaling is likely responsible for angiogenesis in HUVECs (Figure 5E). We thus performed western blot to examine whether overexpression of miR199b-5p affects the expression of signaling proteins involved in Jagged1/Notch1 signaling. 


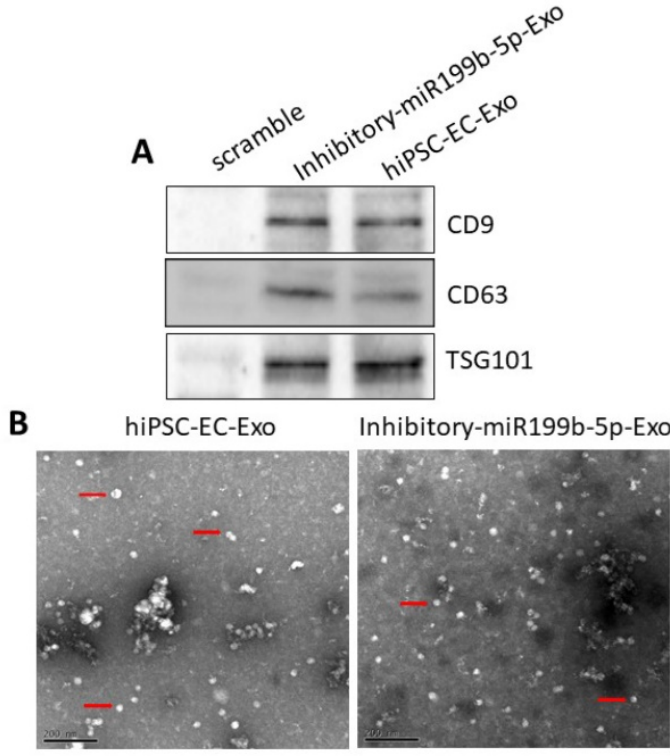

C
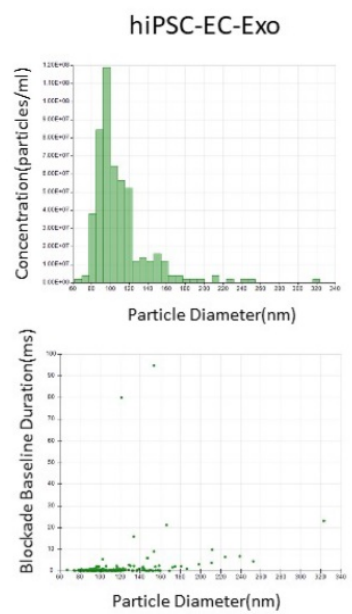

Particle Diameter(nm)
Inhibitory-miR199b-5p-Exo
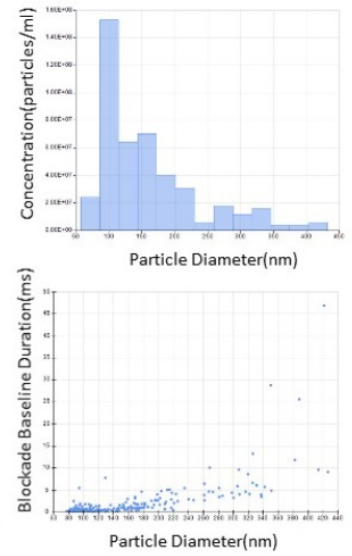

E

D

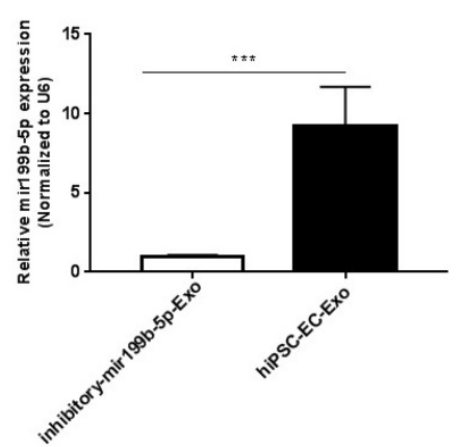

$\mathbf{F}$

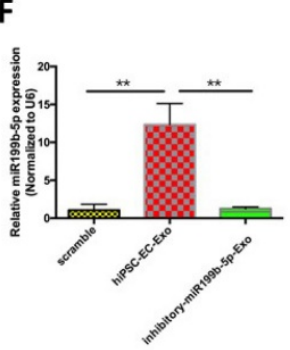

G

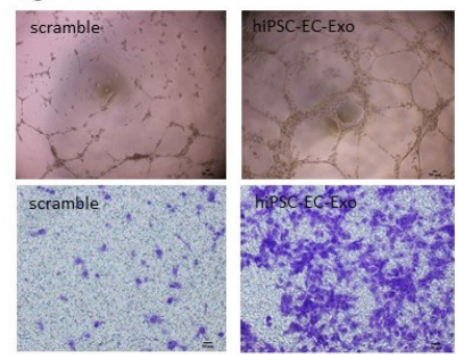

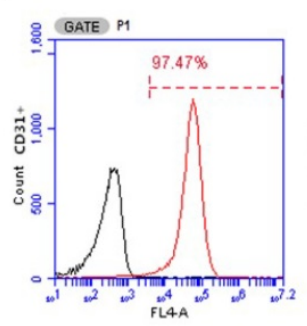

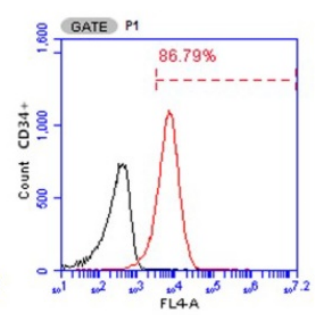

H
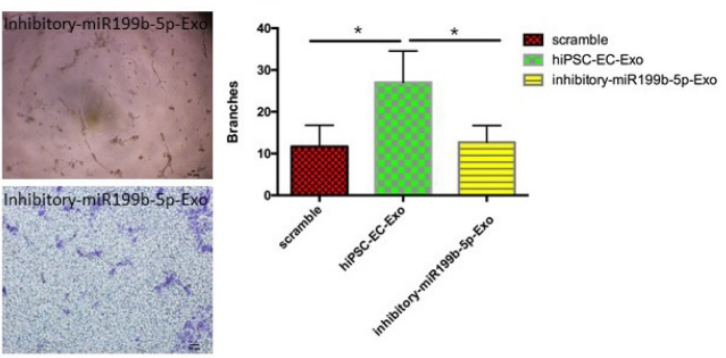

I

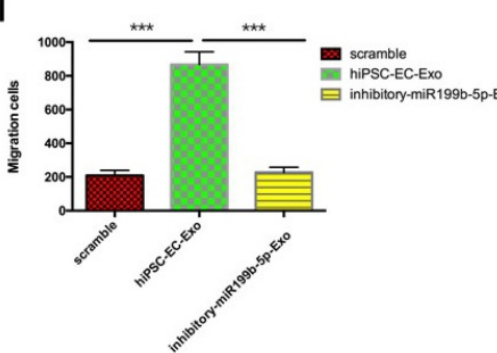

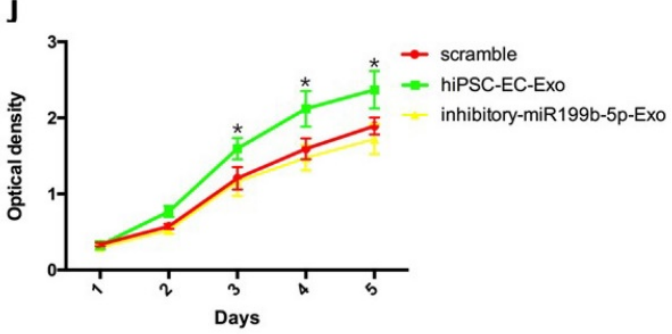

HUVECS

Figure 3. Exosomes derived from iPSC-ECs promote cell migration and proliferation. (A): Exosomes purified from iPSC-ECs were verified by detecting exosome markers, including CD9, CD63 and TSG101 by western blot; (B\&C) SEM and Nanosight were performed to observe the morphology of exosomes and measure the size of exosomes, respectively; (D): Transcriptional levels of miR-199b-5p in iPSC-ECs and inhibitory iPSC-ECs; (E): Immunostaining of iPSC-EC-associated markers (CD31 and CD34); (F) Transcriptional levels of miR-199b-5p in HUVECs coculture with exosomes enriched miR-199b-5p or inhibitory miR-199b-5p were examined by qPCR; (G): Endothelial tube formation of HUVECs, co-cultured with either iPSC-ECs or inhibitory- iPSC-ECs, was captured by light microscopy (original magnification, $\times 100)$; Photo images of Transwell analysis of HUVECs co-cultured with either iPSC-ECs or inhibitory- iPSC-ECs; $(\mathbf{H})$ The tube-like structures were determined by pixel density, values are mean \pm SD, * $<<0.05$; $(\mathbf{I})$ Migration rates of HUVECs co-cultured with either iPSC-ECs or inhibitory- iPSC-ECs, results are presented as mean \pm SD, * $p<0.05$; (J): Growth curves of HUVECs co-cultured with either iPSC-ECs or inhibitory- iPSC-ECs were assessed by CCK-8 assay. The OD value at $450 \mathrm{~nm}$ represents the viable cell number. Results are presented as mean \pm SD, * $\mathrm{p}<0.05$. 
A
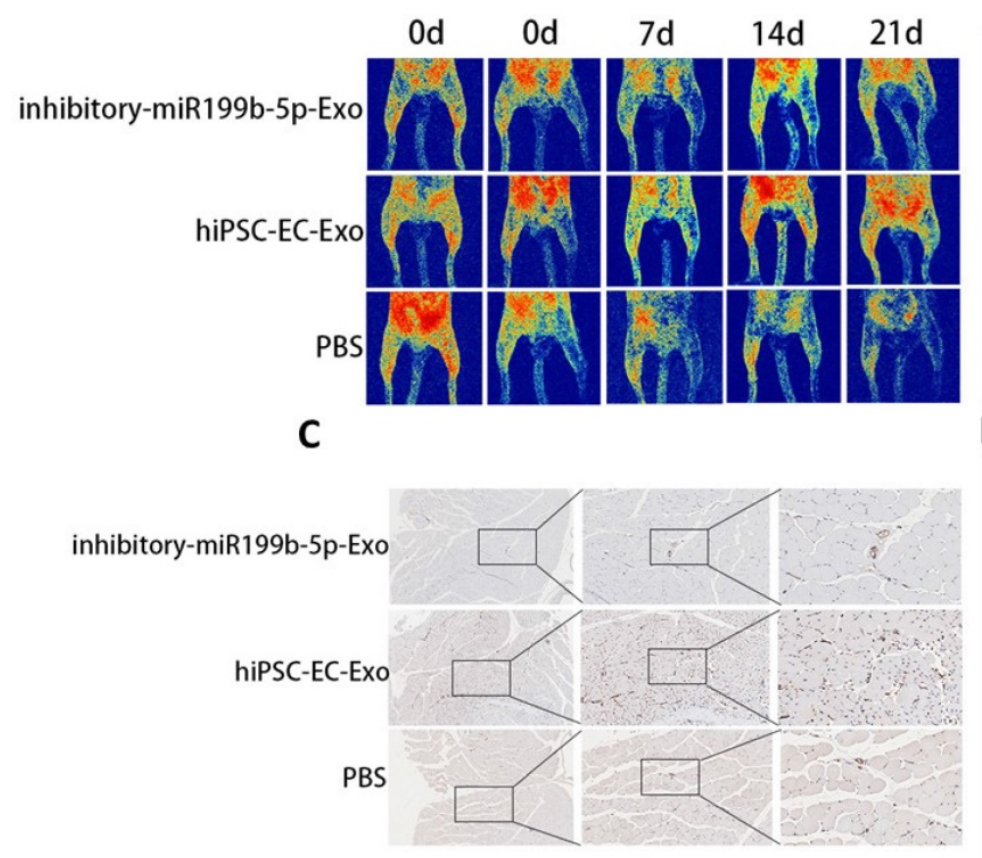

B

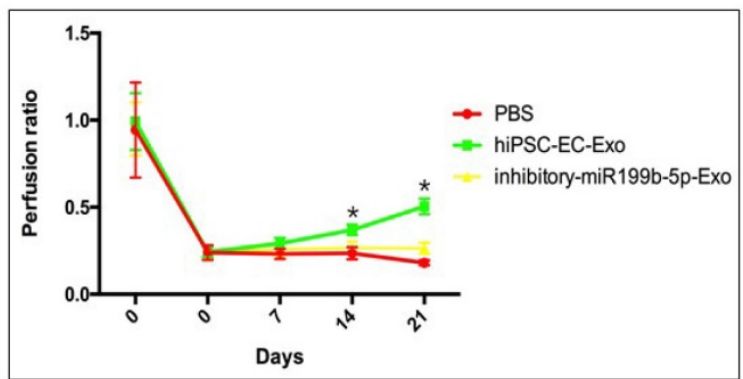

D

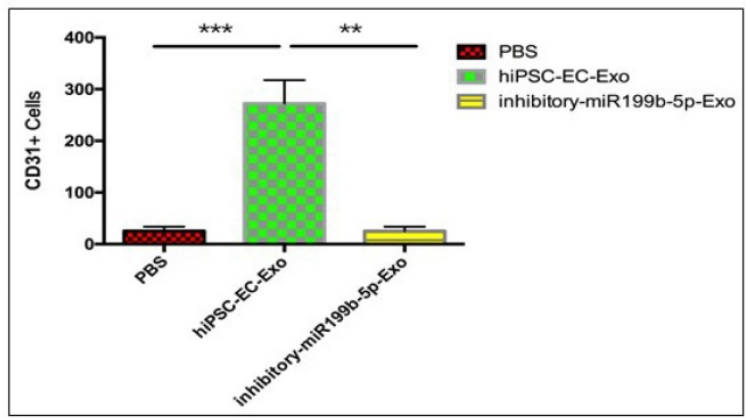

Figure 4. Injection of iPSC-EC-Exo promotes angiogenesis of ischemic limbs. (A): Typical perfusion images of mice that have ischemic limbs were taken by laser speckle contrast imaging. Blood flux is represented by a color scale with red representing the highest flux and dark blue the lowest; (B) Representative curves of perfusion ratio vs time (in days) after initiation of the experiments. The time points are plotted on the $y$-axis against perfusion ratio on the $\mathrm{x}$-axis. Results are presented as mean $\pm \mathrm{SD}, * \mathrm{p}<0.05$. (C\&D) Immunohistochemical staining of muscle tissues from CD31. Three independent sections with staining of CD31 were captured and CD31 positive cells were counted for statistical analysis. Scale bar $=50 \mu \mathrm{m}$.

Figure 5F\&H found that HUVECs expressing miR-199b-5p have a significantly decreased protein level of Jagged1, while HUVECs expressing inhibitory miR-199b-5p have a significantly increased protein level of Jagged1 as compared to wild-type HUVECs. As a target gene of Jagged1/Notch signaling, transcriptional suppressor HES1 was also found to be downregulated by miR-199b-5p (Figure 5G\&J). Consequently, we observed that the protein expression level of VEGFR2 was significantly increased in HUVECs expressing miR-199b-5p, but significantly decreased in HUVECs expressing inhibitory-miR-199b-5p (Figure 5F\&I). These results together indicate that VEGFR2 expression is negatively associated with Jagged1 and HES1 expression, and miR-199b-5p likely regulates VEGFR2 via inhibition of Jagged1/Notch1 signaling. Finally, we cocultured HUVECs with exosomes derived from hiPSC-EC-Exo and inhibitory hiPSC-ECExo, respectively, as above-mentioned. The protein levels of Notch-1, Jagged-1, HES1 and VEGFR2 in HUVECs were evaluated by western blot. Similarly, we found that HUVECs cocultured with hiPSC-ECExo have significantly decreased protein levels of Notch1, Jagged1 and HES1, but increased protein level of VEGFR2 as compared to wild-type HUVECs. Conversely, HUVECs cocultured with inhibitory hiPSC-EC-Exo have profoundly increased protein levels of Notch1, Jagged1 and HES1 but a decreased protein level of VEGFR2 as compared to wild-type HUVECs (Figure 5K-P). Collectively, these results highly indicate that exosomes derived from iPSC-EC likely serve as a paracrine factor, contributing to the regulation of angiogenesis.

\section{Discussion}

iPSC-ECs have recently emerged as an attractive cell type for treating myocardial ischemia and peripheral vascular disease ${ }^{22,23}$. There are 2 advantages in using iPSC-ECs as a regenerative therapy: (1) iPSC-ECs are customized and infinitely expandable $e x$ vivo, therefore offer an unlimited source for EC generation; (2) patient-specific, iPSC-derived ECs can be used for autologous transplantation without the necessity for immunosuppression. However, the biological function and therapeutic potential of iPSC-ECs remain to be evaluated. In this study, we demonstrated that a significant component of the proangiogenic paracrine activity associated with iPSC-ECs is mediated by exosomes. The exosomes derived from iPSC-ECs were morphologically similar in size and shape to typical exosomes described previously and carried well-known exosome protein markers. Functionally, we found that exosomes derived from iPSC-ECs have the potentials to promote neovascularization both in vitro and in vivo. 

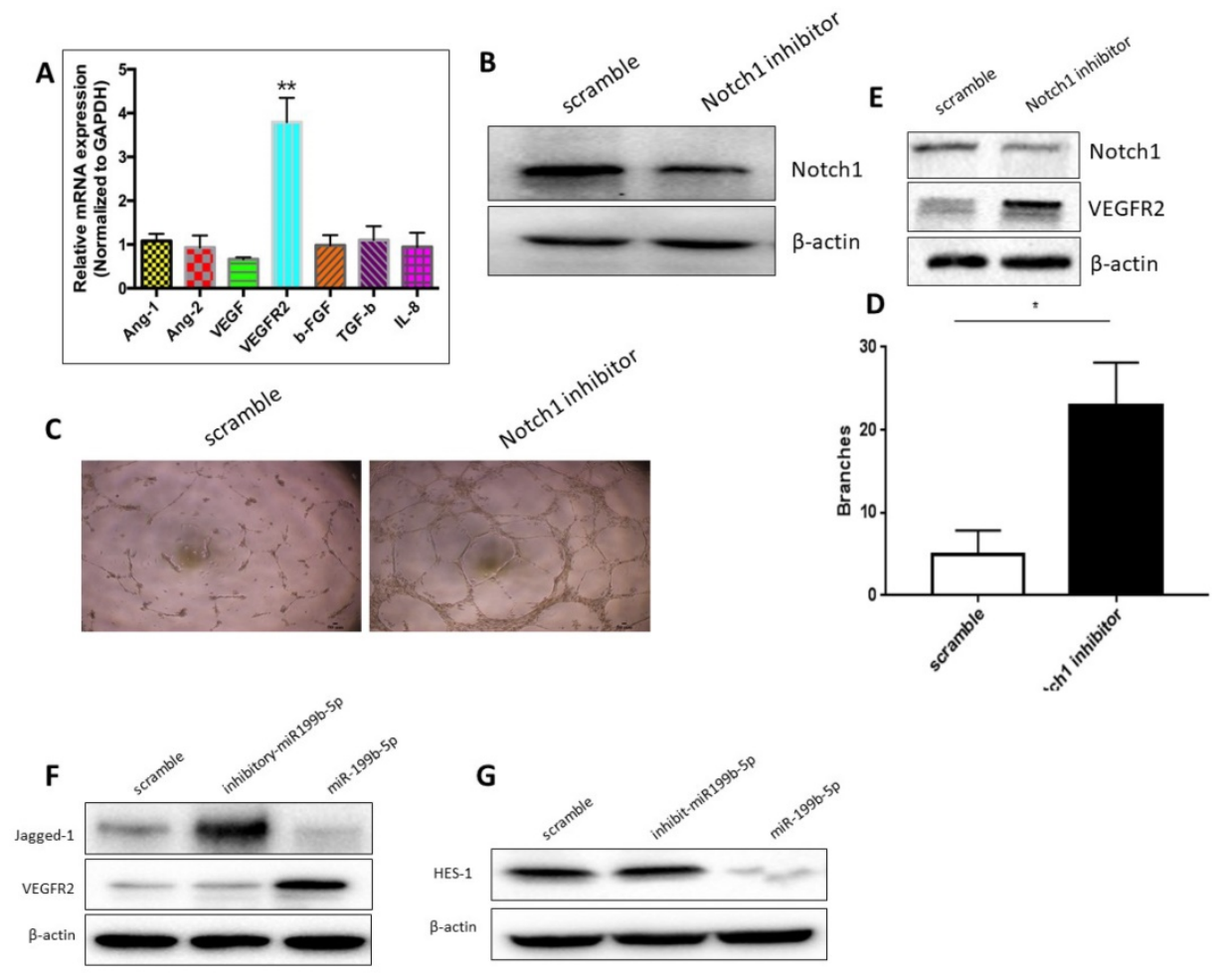

H

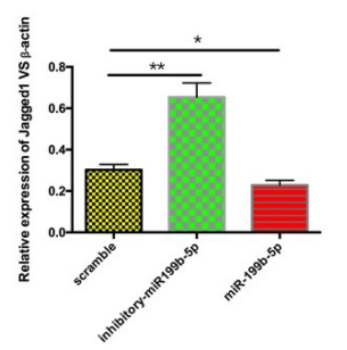

I
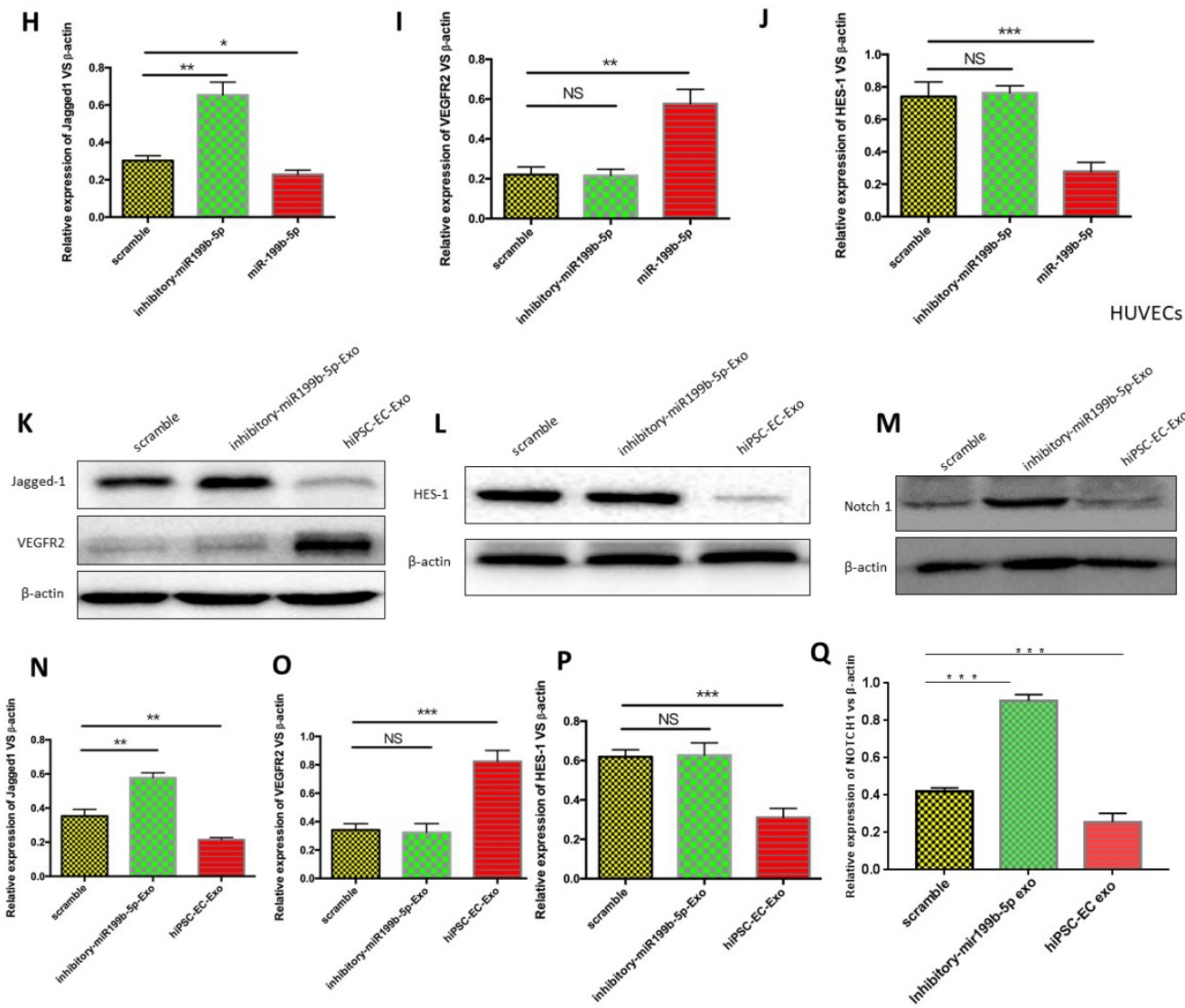

Figure 5. The mechanism by which miR-199b-5p promotes angiogenesis of ischemic limbs. (A): Expression profiles of angiogenic genes (Ang-1, Ang-2, VEGF, VEGFR2 $\beta$-FGF. TGF- $\beta$ and IL-8) from HUVECs expressing either miR-199b-5p or inhibitory-miR-199b-5p were examined by qPCR; (B): Notch1 inhibitor DAPT 75umol was applied for HUVECs to block the activation of Notchl. Western Blot was performed to determine the protein expression of Notchl and $\beta$-actin. (C): Endothelial tube formation of HUVECs co-cultured with either DMSO or DAPT, was captured by light microscopy (original magnification, $\times 100$ ). (D) The tube-like structures were determined by pixel density, values are mean $\pm S D$, * $p<0.05$; (E): Western blot was performed to determine the protein expression levels of VEGFR2 in HUVECs treated with either DMSO or DAPT. (F\&G): Western blot was performed to determine the protein expression levels of Jagged-1, HES-1 and VEGFR2 in HUVECs expressing either miR-199b-5p or inhibitory-miR-199b-5p; (H-J): Protein levels of Jagged-1, VEGFR2 and HES-1 were quantitated by densitometric analysis using NIH Image], normalized to $\beta$-actin and presented as mean \pm SD, * $p<0.05$; (K-Q): HUVECs cocultured either with iPSC-ECs or inhibitory- iPSC-ECs were harvested and protein levels of Notch 1, Jagged-1, VEGFR2, HES-1 and $\beta$-actin were examined by western blotting and quantitated by densitometric analysis using NIH Image, normalized to $\beta$-actin and presented as mean $\pm S D$, * $p<0.05$. 


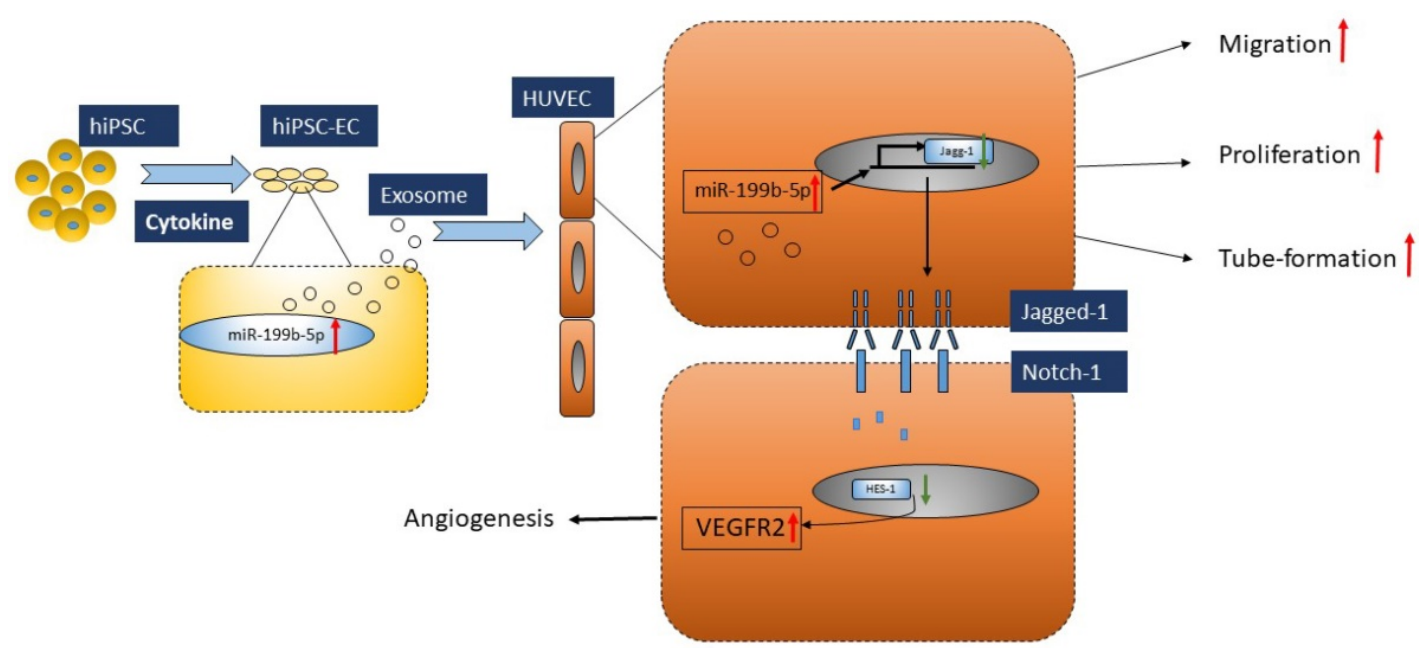

Figure 6. Proposed model of regulatory role of miR-199p-5b in HUVEC angiogenesis. Exosomes derived from iPSC-EC supports HUVEC migration, tube-formation and proliferation via miR-199p-5b. miR-199p-5b is a modulator of VEGFR2 expression via transcriptional suppressor HES-1. Green arrows indicate down-regulation and red arrows indicate up-regulation.

Cell-cell communication can occur by several means, including chemical receptor-mediated events, direct cell-cell contact and cell-cell synapses ${ }^{24,}{ }^{25}$. However, the transfer of exosome-derived miRNAs to recipient cells is a novel mechanism allowing gene communication between mammalian cells ${ }^{26,27,28}$. It is reported that exosome miRNAs, such as miR-1, miR-17, miR-18, miR-181 and miR-375 play a central role in regulating distinct biological processes, which include angiogenesis ${ }^{29,30,31}$. In the context of vascular disease, the miRNA family containing miR-199a-1, miR-199a-2 and miR-199b are potentially important ${ }^{18,}$ 32,33 . Evidence shows that miR-199b is modulated in cardiomyocytes and ECs by exposure to hypoxia and high glucose in vitro. More recently, it was demonstrated that miR-199b is involved in the process of EC differentiation from iPSCs by regulating VEGF expression under transcriptional control of STAT3 ${ }^{17}$. Similarly, our study first provided evidence that miR-199b-5p was significantly upregulated during the process of iPSC-EC differentiation, which renders iPSC-ECs a unique potential as a regenerative therapeutic agent for ischemia diseases.

During postnatal growth, the size and transport capacity of the vasculature need to expand significantly. Angiogenesis, the expansion of a pre-existing vessel network via a combination of sprouting, proliferation, and remodeling process, predominates in most settings, especially during tumor development and ischemic diseases ${ }^{34,35}$. As one of the closely related regulatory mechanisms of angiogenesis, notch signaling has been demonstrated to be essential for the formation and maintenance of the vascular system in several genetic mouse systems ${ }^{36,37}$. Notch signaling is a highly conserved pathway that is regulated by interactions between trans-membrane ligands and notch receptors. Recently, target prediction databases and literature searches highlighted that Jagged 1, one of the ligands of Notch receptors expressed in vasculature, is an additional target of miR-199b, and this finding was verified by demonstrating that miR-199b-5p serves as a miRNA suppressor of Jagged1 in ovarian cancer ${ }^{38,39}$. In line with the above studies, our study found that overexpression of miR-199b-5p results in the enhancement of VEGFR2 expression via inhibition of Jagged-1 mediated signaling. Molecularly, we further demonstrated that upregulation of VEGFR2 is probably regulated via HES1, a transcriptional suppressor involved in the maintenance of stem cells. This is the first evidence that Notch-mediated VEGFR2 upregulation is linked with miR-199b-5p, suggesting that miR-199b-5p participates in regulating angiogenesis in a highly differential manner through the Notch pathway.

In conclusion, we demonstrated that iPSC-EC-Exo enriched with miR-199b-5p has a therapeutic functional effect on promoting profound angiogenesis in a mouse model bearing ischemic limbs. Mechanically, this modulation of angiogenesis is executed via miR-199b-5p-mediated VEGFR2 overexpression (Figure 6). Given that exosomes moderate notch signaling in a paracrine manner through the transfer of miR-199b-5p, essentially regulating angiogenesis, here we provided the first evidence that exosomes derived from iPSC-ECs is a promising therapeutic approach for ischemic diseases.

\section{Acknowledgements}

This study was supported by the National Natural Science Foundation of China (81670442 to LZ and 81700421 to HD). 


\section{Authors Contribution}

HQ, MY, and LZ designed the studies. HQ, MY, $X G, Q N, J C, M L, Y Z$, and GX performed the experiments. HD and $\mathrm{LZ}$ wrote and revised the manuscript.

\section{Competing Interests}

The authors have declared that no competing interest exists.

\section{References}

1. Norgren L, et al. Inter-Society Consensus for the Management of Peripheral Arterial Disease (TASC II). European journal of vascular and endovascular surgery : the official journal of the European Society for Vascular Surgery 2007, 33 Suppl 1: S1-75.

2. Minar E. Critical limb ischaemia. Hamostaseologie 2009, 29(1): 102-109.

3. Rowe VL, Lee W, Weaver FA, Etzioni D. Patterns of treatment for peripheral arterial disease in the United States: 1996-2005. Journal of vascular surgery 2009, 49(4): 910-917.

4. Takahashi K, Yamanaka S. Induction of pluripotent stem cells from mouse embryonic and adult fibroblast cultures by defined factors. Cell 2006, 126(4): 663-676

5. Hu GW, et al. Exosomes secreted by human-induced pluripotent stem cell-derived mesenchymal stem cells attenuate limb ischemia by promoting angiogenesis in mice. Stem cell research \& therapy 2015, 6: 10 .

6. Liu X, et al. Exosomes Secreted from Human-Induced Pluripotent Stem Cell-Derived Mesenchymal Stem Cells Prevent Osteonecrosis of the Femoral Head by Promoting Angiogenesis. International journal of biological sciences 2017, 13(2): 232-244.

7. Wang Y, et al. Exosomes/microvesicles from induced pluripotent stem cells deliver cardioprotective miRNAs and prevent cardiomyocyte apoptosis in the ischemic myocardium. International journal of cardiology 2015, 192: 61-69.

8. Mathiyalagan P, et al. Angiogenic Mechanisms of Human CD34(+) Stem Cell Exosomes in the Repair of Ischemic Hindlimb. Circulation research 2017, 120(9): 1466-1476.

9. Valadi $\mathrm{H}$, et al. Exosome-mediated transfer of mRNAs and microRNAs is a novel mechanism of genetic exchange between cells. Nature cell biology 2007, 9(6): 654-659.

10. Mathivanan S, Ji H, Simpson RJ. Exosomes: extracellular organelles important in intercellular communication. Journal of proteomics 2010, 73(10): 1907-1920.

11. Icli B, et al. MicroRNA-26a regulates pathological and physiological angiogenesis by targeting BMP/SMAD1 signaling. Circulation research 2013, 113(11): 1231-1241

12. Urbich $\mathrm{C}$, et al. MicroRNA-27a/b controls endothelial cell repulsion and angiogenesis by targeting semaphorin 6A. Blood 2012, 119(6): 1607-1616.

13. Fiedler $\mathrm{J}$, Thum $\mathrm{T}$. New Insights Into miR-17-92 Cluster Regulation and Angiogenesis. Circulation research 2016, 118(1): 9-11.

14. Newton WC, Kim JW, Luo JZQ, Luo L. Stem cell-derived exosomes: a novel vector for tissue repair and diabetic therapy. Journal of molecular endocrinology 2017, 59(4): R155-R165.

15. Yuan MJ, Maghsoudi T, Wang T. Exosomes Mediate the Intercellular Communication after Myocardial Infarction. International journal of medical sciences 2016, 13(2): 113-116.

16. Min PK, Chan SY. The biology of circulating microRNAs in cardiovascular disease. European journal of clinical investigation 2015, 45(8): 860-874.

17. Chen T, et al. MicroRNA-199b Modulates Vascular Cell Fate During iPS Cell Differentiation by Targeting the Notch Ligand Jagged1 and Enhancing VEGF Signaling. Stem cells 2015, 33(5): 1405-1418.

18. Du P, et al. Role of miR-199b-5p in regulating angiogenesis in mouse myocardial microvascular endothelial cells through HSF1/VEGF pathway. Environmental toxicology and pharmacology 2016, 47: 142-148.

19. Lv L, et al. Long Noncoding RNA uc001pwg.1 Is Downregulated in Neointima in Arteriovenous Fistulas and Mediates the Function of Endothelial Cells Derived from Pluripotent Stem Cells. Stem cells international 2017, 2017: 4252974.

20. Lv L, et al. Zinc finger protein 191 deficiency attenuates vascular smooth muscle cell proliferation, migration, and intimal hyperplasia after endovascular arterial injury. Journal of vascular surgery 2014, 59(2): 500-509.

21. Zhang L, Zhou M, Qin G, Weintraub NL, Tang Y. MiR-92a regulates viability and angiogenesis of endothelial cells under oxidative stress. Biochemical and biophysical research communications 2014, 446(4): 952-958.

22. Zhou Y, et al. Induced pluripotent stem cell transplantation in the treatment of porcine chronic myocardial ischemia. The Annals of thoracic surgery 2014, 98(6): 2130-2137.

23. Rufaihah AJ, et al. Endothelial cells derived from human iPSCS increase capillary density and improve perfusion in a mouse model of peripheral arterial disease. Arteriosclerosis, thrombosis, and vascular biology 2011, 31(11): e72-79.
24. Roberts CT, Jr., Kurre P. Vesicle trafficking and RNA transfer add complexity and connectivity to cell-cell communication. Cancer research 2013, 73(11): 3200-3205.

25. Brucher BL, Jamall IS. Cell-cell communication in the tumor microenvironment, carcinogenesis, and anticancer treatment. Cellular physiology and biochemistry : international journal of experimental cellular physiology, biochemistry, and pharmacology 2014, 34(2): 213-243.

26. Prathipati P, Nandi SS, Mishra PK. Stem Cell-Derived Exosomes, Autophagy, Extracellular Matrix Turnover, and miRNAs in Cardiac Regeneration during Stem Cell Therapy. Stem cell reviews 2017, 13(1): 79-91.

27. Yang $\mathrm{F}$, et al. Exosomal miRNAs and miRNA dysregulation in cancer-associated fibroblasts. Molecular cancer 2017, 16(1): 148.

28. Bach DH, Hong JY, Park HJ, Lee SK. The role of exosomes and miRNAs in drug-resistance of cancer cells. International journal of cancer 2017, 141(2): 220-230.

29. Stahlhut C, Suarez Y, Lu J, Mishima Y, Giraldez AJ. miR-1 and miR-206 regulate angiogenesis by modulating VegfA expression in zebrafish. Development 2012, 139(23): 4356-4364.

30. Kuhnert F, Kuo CJ. miR-17-92 angiogenesis micromanagement. Blood 2010, 115(23): 4631-4633.

31. Landskroner-Eiger S, Moneke I, Sessa WC. miRNAs as modulators of angiogenesis. Cold Spring Harbor perspectives in medicine 2013, 3(2): a006643.

32. Ghosh A, et al. MiRNA199a-3p suppresses tumor growth, migration, invasion and angiogenesis in hepatocellular carcinoma by targeting VEGFA, VEGFR1, VEGFR2, HGF and MMP2. Cell death \& disease 2017, 8(3): e2706.

33. Liu GT, et al. CCL5 promotes vascular endothelial growth factor expression and induces angiogenesis by down-regulating miR-199a in human chondrosarcoma cells. Cancer letters 2015, 357(2): 476-487.

34. Sanada F, et al. Gene-Therapeutic Strategies Targeting Angiogenesis in Peripheral Artery Disease. Medicines 2018, 5(2).

35. Chung AS, Lee J, Ferrara N. Targeting the tumour vasculature: insights from physiological angiogenesis. Nature reviews Cancer 2010, 10(7): 505-514.

36. Li JL, Harris AL. Notch signaling from tumor cells: a new mechanism of angiogenesis. Cancer cell 2005, 8(1): 1-3.

37. Benedito R, et al. The notch ligands Dll4 and Jagged1 have opposing effects on angiogenesis. Cell 2009, 137(6): 1124-1135.

38. Liu MX, et al. Epigenetic silencing of microRNA-199b-5p is associated with acquired chemoresistance via activation of JAG1-Notch1 signaling in ovarian cancer. Oncotarget 2014, 5(4): 944-958.

39. $\mathrm{Qu} \mathrm{X}$, et al. MiR-199b-5p inhibits osteogenic differentiation in ligamentum flavum cells by targeting JAG1 and modulating the Notch signalling pathway. Journal of cellular and molecular medicine 2017, 21(6): 1159-1170. 